\title{
Invited Papers
}




\title{
INTRODUCTORY REMARKS
}

\author{
J. SAHADE \\ C.C. 677 , Observatorio Astronómico, \\ 1900 La Plata, and \\ C.C. 5, Instituto Argentino de Radioastronomía, \\ 1894 Villa Elisa (Bs. As.); \\ Member of the Carrera del Investigador Científico, \\ CONICET, Argentina.
}

Yoji has asked me to open this Symposium on Evolutionary Processes in Interacting Binary Stars by giving a review on "known species of interacting binaries", but, what can I say that you do not know already? If you permit me, I would like to take the opportunity to just put together a few facts of life that came to my mind when thinking on how to fulfil my assignment. I do not expect to say anything new, though.

1. Perhaps $I$ ought to start by emphasizing the we 11 known fact that in the evolutionary processes in interacting binary stars, broadly speaking, there is a stage of rapid mass loss which is followed by a stage of slow mass loss. It is in the latter stage that we find the groups in which the close binary systems sppear to sort out. These well known groups are those of

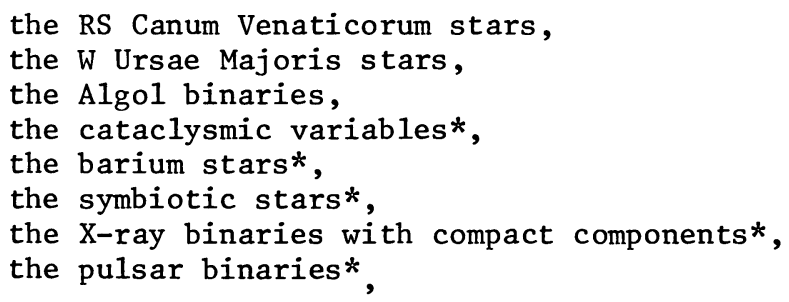

and we should perhaps insert somewhere in this listing the unevolved and the $\zeta$ Aurigae binaries. The order is, of course, somewhat arbitrary in the sense that it does not imply necessarily an evolutionary sequence, although it may involve some age sequence of the component stars.

The next characterization of these groups is that some of them are apparently undergoing mass loss for the first time in their lives, after the "main sequence" stage, and others, those that are singled out with an asterisk, are undergoing at least a second episode of mass loss.

This latter conclusion comes about because of the fact that in all cases the component that is accreting mass or towards which the flow of 
the mass being shed by one of the components of the system is directed, is a compact object, either a white dwarf or a neutron star, that is, a star that is well advanced in its evolution.

2. Another fact of life is that the process of rapid mass loss appears to produce very bizarre objects. In 1986 we (Sahade 1986a) suggested that peculiar binaries are most probably in the evolutionary stage of rapid mass loss, immediately preceding or following the mass-ratio rever sal episode. In addition, we (Sahade 1987, 1988a,b,c) further suggested a working hypothesis for the evolution of the gaseous structure that characterizes these objects, through the stage of rapid mass loss.

It would seem as though peculiar interacting binaries hold the information that could answer the question as to how much mass is lost in the process of rapid mass loss, to how the process accurs, and to how much mass is lost to the system, questions that are still pending of an answer.

The group we (Sahade $1966,1986 \mathrm{~b}$ ) suggested in 1963 and in 1985 appears actually to be formed by objects which are past the stage of mass-ratio reversal but not yet in what we would call the slow mass loss process.

3. It has long been recognized that mass loss in a close binary system occurs either by Roche low overflow or through the stellar wind. As a consequence, in the case of systems with early-type components, evolution ought to proceed somewhat differently than in the cases of systems with no early-type components.

Another possible mechanism for "mass transfer" in interacting binaries, that appears to be relevant in the case of evolved systems like the cataclysmic variables, and was first suggested by Kraft, Mathews and Greenstein (1962), appears to be provided by gravitational radiation (cf. Paczyński 1967; Faulkner 1971; Rappaport, Joss and Webbink 1982).

3a. The effect of mass loss through the Roche lobe overflow shows in the presence of a "gas stream" which gives rise to a "circumstellar envelope" around the companion, and also to a "circumbinary envelope". The former could be optically thin or optically thick, depending on the relative size of the companion to the star that loses mass (cf. Lubow and Shu 1975). The circumstellar envelope is usually designated now an "accretion disk".

In regard to the gaseous stream, let me remind you that in the early interpretation of $\beta$ Lyrae, two streams were suggested, each one arising in one of the components, but later it was shown that there is actually only one stream in the system, and such is the case in every interacting binary for which the "classical" picture holds. The stream always goes from the larger, less massive component towards the smaller, more massive companion.

$3 b$. The effect of the stellar winds in early-type systems is to produce a gaseous medium in which the two components are embedded. If the two components are early-type objects then we should observe the effects of wind collision. 
The literature is recording now at least four papers where the finding of such an effect is claimed, but the story actually started with Huang and Weigert's (1982) paper where they considered the "the bow wave shocks that result from the interaction of two stellar winds" in a wide binary system like $\alpha$ Scorpii "containing a late supergiant and an early main sequence star (B2.5 V)". The conclusion was that the emission of [Fe II] that is observed about 1 " west of the B star probably arises in the front.

Shortly after, Wallerstein et al. (1984) and Willson et al. (1984) tried to explain the emission profiles of two symbiotic objects, V1016 Cygni and HM Sagittae, and concluded: 1) that they "could result from the roughly conical interaction nebula produced by the collision of two stellar winds"; 2) that the X-ray emission originates in "an optically thin region between the two stars in the system where the winds collide head on".

Although these earliest theoretical papers did not involve earlytype objects, it was natural to look first for the effect in early-type binaries.

Wallerstein et al.'s (1984) and Willson et al.'s (1984) papers were invoked by Brandi, Ferrer and Sahade (1989) as providing a good explanation for the existence of a concentration of matter between the two components of the Wolf-Rayet binary $\gamma_{2}$ Velorum (WC8+08 I), which was necessary to introduce when trying to interpret the profile variations in the IUE spectrum of the object. As a consequence of this paper, a search for a similar effect in the early-type binaries HD 47129 (08 V+ ) and AO Cassiopeiae (09 III + 09 III) was carried out successfully by Sahade and Brandi (1991).

An earlier paper on detection of wind collission effects was due to Shore and Brown (1988) who did interpret dramatic profile variations in He II 1640 and in the resonance lines of CIV in the IUE spectrum of the Wolf-Rayet binary V444 Cygni (06-8 III+WN4) as resulting from wind collision effects. Another approach has been followed by Gies and Wiggs (1991) who analyzed the difference profiles of $\mathrm{H} \alpha$ and He I 6678 in AO Cas, after substracting model photospheric profiles, described by Mochnacki and Doughty (1972), from the observed ones. Gies and Niggs (1991) are undergoing similar work with l Orionis (09 III), 29 UW Canis Majoris $(07 f+07)$ and HD 47129.

The systems AO Cas and $\mu^{1}$ Scorpii have been observed with the IUE during 60 consecutive hours to try to understant better the effects of wind collision and the origin of the "discrete absorption components".

More work should be done in regard to the problem of wind interaction and we should try to profit from the conclusions by Girard and Willson (1987).

In early-type binaries the "classical" Roche equipotentials, the contact surfaces, no longer exist (cf. Sahade and Wood 1978 and referen ces therein), and, as a consequence, the picture of an interacting binary first proposed, theoretically, for the case of B Lyr (Kuiper 1941) and later built on observational results from a number of close pairs, principally by Struve and his coworkers, no longer hold. And this is coherent with what we have said above. 
3c. About three decades ago, we (Sahade 1959) called attention to the fact that the shortest binary periods were found among systems with compact components. Gravitational radiation, the third mechanism of mass loss that we have mentioned, that produces a decay in the orbit and, therefore, a smaller-size system, could provide the explanation (cf. Faulker, 1971). Recently, Eggleton (1986) has reviewed the processes that may shorten or lengthen the period of a binary.

4. From the observations with the IUE satellite we have been able to derive valuable information, but so far only partial, in regard to the temperature and velocity profiles of the gaseous formation in which the systems are embedded.

Early in the game, it was clear that in interacting binaries there are sources of non-thermal energy, and, later, the observations with the IUE satellite disclosed the presence in the spectrum of lines that cannot be accounted for by the radiation field available. In general, we find more than one layer, and as many as three layers, where such lines are formed; one of them close to the region where the gaseous envelope merges with the interstellar medium. And the pattern appears not to be very different from one system to the next.

One of the most interesting results that have emerged from the IUE observations is the fact that the velocities of the gaseous envelope -found to be in expansion from the conventional observations- at some point start to decelerate until they reach a value similar to that of the interstellar medium. This poses the problem as to how mass is actually lost to the system.

4a. Another question that still needs to be answered, is the one that refers to the origin of the X-radiation in non-compact binaries, like Algo1, B Lyr, HD 47129 or AO Cas.

Some people believe that the source in the case of Algol is the corona of the late-type subdwarf component. But in $\beta$ Lyr there is no late-type component, as far as we know. Moreover, it seems difficult to see how the normal atmospheric structure of the late-type component could survive in a configuration such as that of the Algols, and the X-radiation could arise as a consequence of the existence of non-thermal sources of energy that are present in interacting binaries.

In the cases of $\mathrm{HD} 47129$ and $A O$ Cas the X-radiation could originate in the collision of the winds (cf. Willson et al. 1984), but the answer in each case, would only come from observations in $\mathrm{X}$-rays of appropriate systems throughout their orbital cycles.

5. Finally, I should refer to the fact that the importance of interacting binaries has been greatly enhanced by the discovery that these objects might be the source or major sources of cosmic radiation (cf. Weekes 1991). With the use of the Cherenkov radiation technique, it has been detected and confirmed that Cygnus $X-3$, Hercules $X-1$ and Vela $X-1$ are strong emitters of very high energy $\gamma$-rays, of the order of $10^{34}-10^{38}$ ergs $\mathrm{s}^{-1}$, that is, as much energy as the one that is emitted by early type systems in the X-range of energy.

It is most interesting that Cyg X-3 emits strongly not only in the TeV but also in the $\mathrm{PeV}$ and the EeV ranges of energy, while apparently Her $X-1$ and Vel $X-1$ have only been detected in the TeV and PeV's. 
Weekes again gives also a list of other close binaries with neutron star components and even two cataclysmic variables as unconfirmed discoveries. It is clear that we are dealing with non-thermal sources that give rise to fluxes that are abnormally strong, departing drastically from what one would expect from the behaviour elsewhere in the spectrum. The results from the GRO satellite might produce a similar revolution as the UHURU for our understanding of interacting binaries.

Ans now a question arises. Is the very recent announcement (Bailes, Lyne and Shemar 1991) of PSR 1829-10 being accompanied by a small planet, another message, another piece of the puzzle that we will try to put together in this Symposium?. At any rate, it would seem as though the field of interacting binaries is becoming more and more exciting as time goes by and this makes it imperative to push for optical telescopes and for satellites devoted solely to the study of these objects to permit good orbital cycle coverage of them and to plan observations in a rational way.

\section{REFERENCES}

Bailes, M., Lyne, A.G. \& Shemar, S.L. 1991, Nature, 352 311.

Brandi, E., Ferrer, O.E. \& Sahade, J. 1989, Ap. J. 340, 391; Erratum in Ap.J. $3 \underline{\underline{4}} \underline{\underline{?}}, 561,1989$.

Eggleton, P.P. 1986, in The Evolution of Galactic X-Ray Binaries, eds. J.

Truemper, W.H.G. Lewin and W. Brinkmann (Reide1), p. 87.

Faulkner, J. 1971, Ap. J. Letters 1 프, L99.

Gies, D.R. \& Wiggs, M.S. 1991, preprint.

Girard, T. \& Willson, L.A., 1987, A\&A 1 을 $2,247$.

Huang, R.Q. \& Weigert, A. 1982 A\&A $112,282$.

Kraft, R.P., Mathews, J. \& Greenstein, J.L. 1962. Ap. J. $13 \underline{\underline{3}} \underline{\underline{6}}, 312$.

Kuiper, G.P. 1941, Ap. J. 93 3133.

Lubow, S.H. \& Shu, F.H. 1975, Ap. J. 198, 383.

Mochnacki, S.W. \& Doughty, N.A. 1972, MNRAS $15 \underline{\underline{6}}, 51$.

Paczyński, B. 1967, Acta Astr. 17, 287.

Rappaport S., Joss, P.C. \& Webbink, R.F. 1982, Ap. J. 224

Sahade, J. 1959, in Modèles d'Etoiles et Evolution Stellaire, Liège Coll., p. 76.

Sahade, J. 1966, in Stellar Evolution, eds. R.F. Stein and A.G.W Cameron (New York: Plenum Press), p. 449.

Sahade, J. 1986a, in New Insights in Astrophysics, ESA SP-263, ed.E.J. Rolfe, p. 267.

Sahade, J. 1986b, in Highlights of Astronomy, ed. J.P. Swings (Reide1), vol. $\underline{\underline{7}}, 175$.

Sahade, J. 1987, Comments in Ap. $12 \underline{\underline{2}}, 13$.

Sahade, J. 1988a, J. Space Astr. Res. 5,1 .

Sahade, J. 1988b, in Atmospheric Diagnostics of Stellar Evolution, IAU Coll. 108, ed.K. Nomoto (Springer), p. 199.

Sahade, J. 1988c, in Progress and Opportunities in Southern Hemisphere Optical Astronomy, A.S.P. Conference Series, vol.1, eds. V.M. Blanco and M.M. Phillips, p. 84.

Sahade, J. \& Brandi, E. 1991, Ap. J., to appear in the October 1 issue. Sahade, J. \& Wood, F.B. 1978, Interacting Binary Stars (Pergamon), p.14. Shore, S.N. \& Brown, D.N. 1988, Ap. J. 334, 1021.

Wallerstein, G., Willson, L.A., Slazer, J. \& Bruge1, E. 1984, A\&A 1333,137. Weekes, T.C. 1991, preprint of a Chapter for The Realm of Interacting Binaries, eds. J. Sahade, G.E. McCluskey, Jr. and Y. Kondo (Kluwer), in press. 
Willson, L.A., Wallerstein, G., Bruge1, E.W. \& Stence1, R.E. 1984, A\&A 133,154 . 